\title{
Biotic interactions are more important at species' warm vs. cool range-edges: a synthesis
}

\author{
Alexandra Paquette ${ }^{1}$ and Anna Hargreaves ${ }^{1}$ \\ ${ }^{1}$ McGill University
}

April 19, 2021

\begin{abstract}
Predicting which ecological factors constrain species distributions is a fundamental question in ecology and critical to forecasting geographic responses to global change. Darwin hypothesized that abiotic factors generally impose species' high-latitude and high-elevation (typically cool) range limits, whereas biotic interactions more often impose species' low-latitude/low-elevation (typically warm) limits, but empirical support has been mixed. Here, we clarify three predictions arising from Darwin's hypothesis, and show that previously mixed support is partially due to researchers testing different predictions. Using a comprehensive literature review (886 range limits), we find that biotic interactions, including competition, predation, and parasitism, influenced species' warm limits more often than species' cool limits. At cool limits, abiotic factors were consistently more important than biotic interactions, but temperature contributed strongly to cool and warm limits. Our results suggest that most range limits will be sensitive to climate warming, but warm limit responses will depend strongly on biotic interactions.
\end{abstract}

\section{Hosted file}

Paquette \& Hargreaves main 2104 07.pdf available at https://authorea.com/users/408718/ articles/518622-biotic-interactions-are-more-important-at-species-warm-vs-cool-rangeedges-a-synthesis 